\title{
Intra-articular hyaluronic acid and corticosteroids in the treatment of knee osteoarthritis: A meta-analysis
}

\author{
FANG WANG and XIJING HE \\ Orthopedics Department, Second Affiliated Hospital, College of Medicine, \\ Xi'an Jiaotong University, Xi'an, Shaanxi 710004, P.R. China \\ Received January 7, 2014; Accepted June 30, 2014
}

DOI: $10.3892 /$ etm.2014.2131

\begin{abstract}
The aim of the present study was to evaluate the therapeutic effect of intra-articular hyaluronic acid (HA) in comparison to corticosteroids (CS) for knee osteoarthritis (OA). The data sources included PubMed, EMBASE, The Cochrane Central Register of Controlled Trials and hand searched reviews. Randomized controlled trials that reported the effects of intra-articular HA and CS in the treatment of knee OA were selected based on specific inclusion criteria. A meta-analysis was performed for the visual analog scale (VAS), Lequesne index, Knee Society Clinical Rating System (KSS), maximum flexion and adverse events of knee OA. Sensitivity analysis was also conducted to avoid bias. The seven eligible trials included 583 participants and the majority of the trials were of high quality. After one month, the mean difference in the VAS was 1.66 [95\% confidence interval (CI); -0.90, 4.23), indicating equal efficacy for HA and CS. However, after three months, the mean difference was -12.58 (95\% CI; -17.76, -7.40), while after six months, the difference was -9.01 (95\% CI; -12.62, -5.40), favoring HA. For the additional indicators, including the Lequesne index, the KSS, maximum flexion and adverse events, no statistically significant differences were observed between the two treatment approaches for knee OA. Therefore, the results of the meta-analysis highlight a therapeutic trajectory for intra-articular HA in knee OA pain, as compared with CS, over six months post-intervention. After one month, the two approaches exhibited equal efficacy; however, in the long term, HA was found to have an enhanced effect. No statistically significant difference was observed in the adverse events caused by the two interventions. Further investigation and understanding into the trend observed in the present study may aid clinicians in the treatment of knee OA.
\end{abstract}

Correspondence to: Dr Xijing He, Orthopedics Department, Second Affiliated Hospital, College of Medicine, Xi'an Jiaotong University, 157 Xiwu Road, Xi'an, Shaanxi 710004, P.R. China E-mail: xijing_h@vip.tom.com

Key words: osteoarthritis, knee, intra-articular, hyaluronic acid, corticosteroids, meta-analysis

\section{Introduction}

Osteoarthritis (OA) of the knee is a common, chronic joint disorder, characterized by articular cartilage degeneration and secondary hyperosteogeny (1). OA often causes severe pain in the knee joint and affects $35 \%$ of individuals that are $>65$ years-old (2). There are an estimated 46 million (22\%) adults in the USA suffering from OA (3). OA requires a variety of treatments to ease the pain and improve functioning. Currently, several therapeutic approaches are used, including rest, medication, other noninvasive interventions, nonsurgical invasive interventions and surgical interventions (4). However, if pain persists following rest or medication and other noninvasive interventions have failed, then prior to surgical intervention, intra-articular injections of a number of drugs may be administered. These typically include hyaluronic acid (HA), corticosteroids (CS) and diclofenac.

Intra-articular HA injections have been demonstrated to be beneficial in the treatment of OA, improving joint lubrication and synovial fluid viscosity, normalizing hyaluronan synthesis, inhibiting proteoglycan degradation and exhibiting analgesic and anti-inflammatory effects (5-8). However, the safety of HA remains controversial. Several studies (9-13) have revealed that the use of HA may cause an increased risk of serious adverse events and local adverse events, indicating that intra-articular HA injections should be discouraged.

Intra-articular injections of CS have been used for the past decade in the treatment of OA, and appear to be relatively safe. CS have anti-inflammatory effects due to the inhibition of inflammatory cytokines, as well as the inhibition of the pathways that lead to their functioning (14). However, the duration effect of CS is considerably less than the recommended interval between doses (15). Therefore, the short-term effects are acceptable; however, the long-term effects require further investigation.

Numerous systematic reviews have investigated the effects of HA and other placebos $(9,16-18)$, or CS and placebos $(19,20)$; however, there are few studies that have compared HA and CS (15). Thus, the aim of the present study was to conduct a meta-analysis to determine which treatment method was more effective, comparing intra-articular HA injection with $\mathrm{CS}$, and to assess whether intra-articular HA injections were associated with a lower incidence of adverse events compared with CS. 


\section{Materials and methods}

Selection of trials. By performing a comprehensive search based on electronic databases and manual literature searches, all the relevant randomized controlled trials (RCTs) comparing intra-articular injections of HA with CS until July 2013 were identified. The electronic sources included PubMed, EMBASE and The Cochrane Central Register of Controlled Trials. Search terms included 'hyaluronic acid', 'hyaluronan', 'corticosteroids', 'glucocorticoids', 'knee osteoarthritis' and 'randomized controlled trials'. In addition, references of the relevant selected articles were reviewed to identify further articles. There were no language restrictions and no protocol for systematic review.

Articles were included if they met the following criteria: (i) Patients were $\geq 18$ years-old and had symptomatic knee OA; (ii) random allocation of treatments; (iii) compared the therapeutic effects of intra-articular HA with intra-articular CS; (iv) follow-up time was $\geq 3$ months; (v) inclusion of $\geq 1$ valid outcome, including the visual analog scale (VAS), Lequesne index, range of motion of the knee, Knee Society Clinical Rating System (KSS) and adverse events; and (vi) outcomes were expressed as mean values or standard deviations (SD), and graphic outcomes as numerical values.

Articles reporting the result of intramuscular or oral drug interventions were excluded, as well as those whose outcomes compared HA/CS with other placebos.

Data collection and analysis. Two independent authors searched the electronic databases based on the titles and abstracts of all the trials to identify relevant studies for inclusion. The authors independently completed the evaluation of the included trials. When the data were incomplete, the author was contacted for sufficient information. If the article did not meet the inclusion criteria, it was excluded from the analysis If no agreement was reached, the senior author (Dr Xijing He) made the final decision. Data of selected articles were carefully cross-checked to ensure that no duplicate data had been presented.

Data extraction. General characteristics, intervention or treatment methods and outcomes were recorded for each study included in the meta-analysis. General characteristics included the publication year, geographical location, participant number and demographics, follow-up time and study design. For all the included articles, the intervention methods were recorded, including the HA/CS dose regimen and frequency. The measured outcomes, as described in the inclusion criteria, were also observed, and all adverse events were recorded. If necessary, the authors were contacted for further information.

Assessment of trial quality. Two authors independently assessed the methodological quality using the Jadad scoring system (21). The Jadad scoring system scored the trials methodologically according to the principle of randomization (0-2 points), blinding implementation ( $0-2$ points) and the withdrawal and lost to follow-up (0-1 points) of the included studies. Using the Jadad scoring system, studies with a total score of 0-2 points were considered as low quality studies, while a total score of $\geq 3$ points were considered high-quality research. The two authors tried to reach a consensus when there were divergences.

Meta-analysis assessment of the treatment effects and outcomes. A meta-analysis of the data, including the effects and outcomes of the treatments, was performed using the Inverse Variance method with Review Manager software (RevMan version 5.0; Cochrane Collaboration, Freiburg, Germany). For continuous data, such as the VAS, mean \pm SD values were used to calculate the weighted mean difference and $95 \%$ confidence interval (CI). For dichotomous data, relative risk (RR) and 95\% CI were applied.

Assessment of heterogeneity. Statistical heterogeneity was assessed using the $\chi^{2}$ test (22). A value of $\mathrm{I}^{2}>50 \%$ was indicative of substantial heterogeneity, recommending a random effects modeling estimate. Otherwise, a fixed effects approach was used.

Analysis of sensitivity. Reanalyzing the data using different statistical approaches, for example using a random effects model instead of a fixed effects model, was utilized for the sensitivity analysis of the meta-analysis.

\section{Results}

Included studies. An initial search yielded a total of 342 studies, of which 316 were excluded following title and abstract screening. The remaining 26 studies were fully retrieved, and 15 studies were excluded as they did not meet the inclusion criteria. A total of 11 RCTs fit the inclusion criteria (23-33). However, two of the trials assessed the efficacy of intra-articular HA with or without CS $(31,32)$, one study reported only median values of the outcome results and had no mean values or measures of variance (26), while an additional study did not use a validated scale to report the outcomes (33). Therefore, the meta-analysis was performed on seven studies with a total 583 patients. A detailed flow chart for the selection is shown in Fig. 1.

Quality of studies. For all the included studies, the methodological quality represented the risk of bias assessment. All the studies adopted the method of randomization $(23-25,27-30)$ and the use of allocation concealment was unclear with the exception of one study (30). Three studies applied a double-blind technique $(23,28,29)$, while two studies applied a single-blind study design $(24,27)$. Only one study did not use a blinding method (25), indicating a potential selection bias. The quality scores of the included studies are shown in Table I.

Study characteristics. A total of 583 participants (222 males and 361 females) were included from the seven studies. In total, 298 participants were administered an HA arm and 285 participants received CS. Of the seven studies used in the analysis, one compared Hyalgan with triamcinolone hexacetonide (23), one compared Hyalgan with methylprednisolone (24), one compared Orthovisc (Anika Therapeutics, Inc., Woburn, MA, USA) with methylprednisolone (25), one compared Synvisc with triamcinolone hexacetonide (27), one compared Durolane (Q-Med AB, Uppsala, Sweden) with triamcinolone (28), one compared Ostenil (TRB Chemedica AG, Haar/München, 
Table I. Jadad quality scores of the seven studies of knee OA included in the meta-analysis.

\begin{tabular}{|c|c|c|c|c|c|c|c|c|c|c|c|c|}
\hline Study & 1 & 2 & 3 & 4 & 5 & 6 & 7 & 8 & 9 & 10 & 11 & Jadad score \\
\hline Jones 1995 (19) & + & + & $?$ & + & + & - & + & - & - & + & + & $5 / 5$ \\
\hline Frizziero $2002(20)$ & + & + & $?$ & + & - & - & + & + & + & + & + & $3 / 5$ \\
\hline Tasciotaoglu 2003 (21) & + & + & $?$ & + & - & - & - & + & - & + & + & $2 / 5$ \\
\hline Caborn 2004 (23) & + & + & $?$ & + & - & - & + & + & + & + & + & $5 / 5$ \\
\hline Skwara 2009 (Durolane) (24) & + & + & $?$ & + & + & + & - & - & - & + & + & $5 / 5$ \\
\hline Skwara 2009 (Ostenil) (25) & + & + & $?$ & + & + & + & - & - & - & + & + & $5 / 5$ \\
\hline Shimizu 2010 (26) & + & + & + & + & $?$ & $?$ & $?$ & - & - & + & + & $3 / 5$ \\
\hline
\end{tabular}

Numbers 1-11 follow the Pedro format, and the Jadad score was calculated from the different set of criteria. 1, eligibility criteria specified; 2, patients were randomized to the groups; 3 , concealment of allocation; 4 , groups were similar at the baseline; 5 , patients were blinded; 6 , practitioners administering the intervention were blinded; 7 , assessors were blinded; 8 , measurements of the key outcomes obtained from $>85 \%$ of patients; 9 , intention to treat analysis; 10 , statistical comparisons between groups; 11 , point measures and measures of variability provided; +, criteria clearly satisfied; -, criteria not clearly satisfied; ?, unclear whether criteria was satisfied.

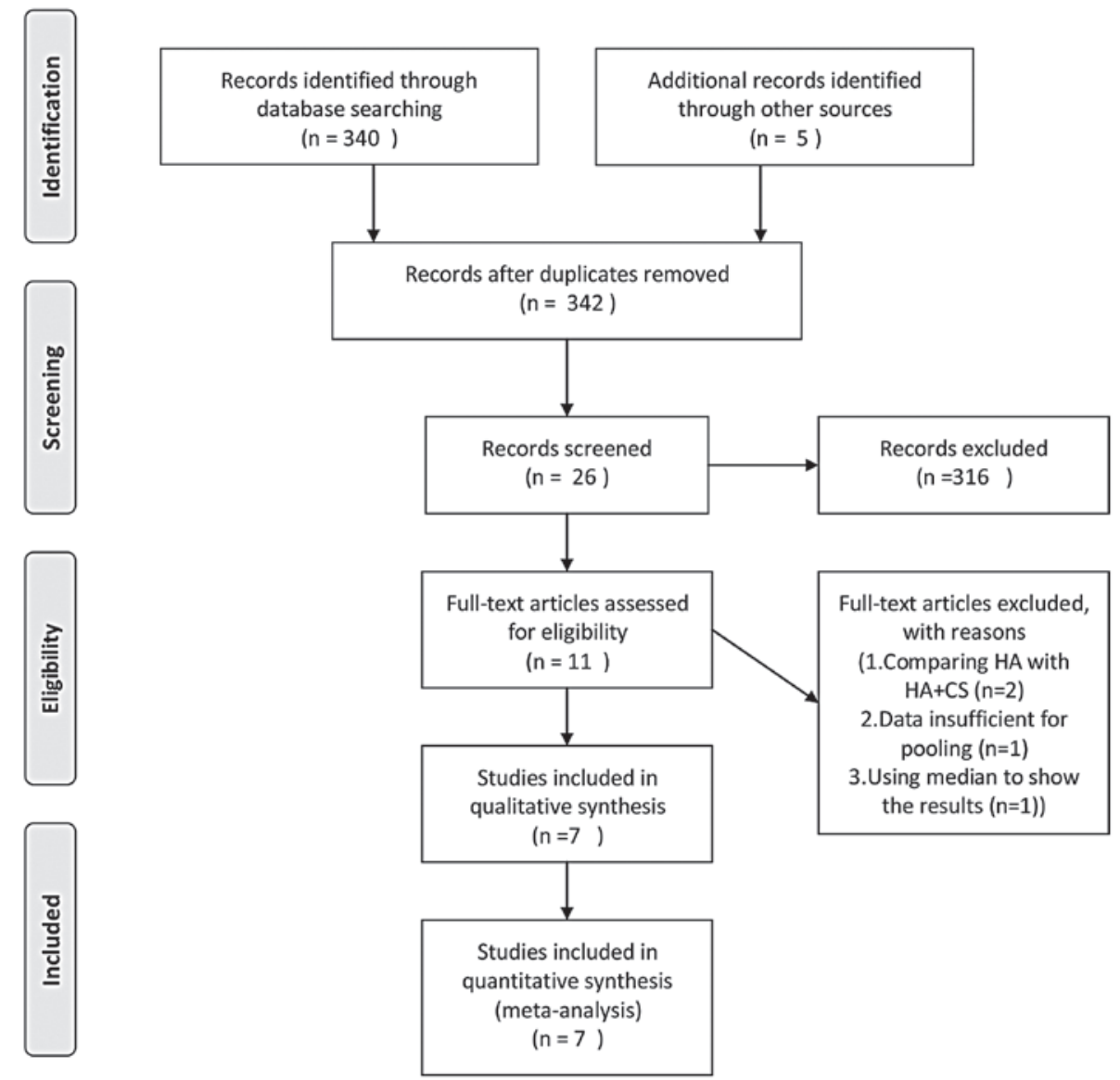

Figure 1. Summary of the search results and the selection procedure for inclusion.

Germany) with triamcinolone (29) and the final study compared Na-HA (Artzdispo; Kaken Pharmaceutical, Tokyo, Japan) with Decadron (30). Details of the studies are shown in Table II.

\section{Meta-analysis}

VAS of knee OA. A total of four studies (23-25,30), including $129 \mathrm{HA}$ and $116 \mathrm{CS}$-treated patients $(\mathrm{n}=245)$, reported detailed data on the VAS score of knee OA after one month of treatment. CS injections were found to reduce the VAS score by a mean of $1.66 \mathrm{~mm}$ compared with the HA group; however, the difference was not statistically significant (95\% CI, -0.90, 4.23; $\mathrm{P}=0.20 ; \mathrm{I}^{2}=48 \%$; Fig. 2).

A total of three studies $(25,27,28)$, including 165 HA and 155 CS-treated patients $(n=320)$, reported detailed data on the VAS score of knee OA after three months. HA injections significantly reduced the VAS score by a mean of $12.58 \mathrm{~mm}$, ranging between 7.40 and 17.76 , when compared with that in the CS group (95\% CI; $\mathrm{P}<0.00001 ; \mathrm{I}^{2}=42 \%$; Fig. 2). 
Table II. Characteristics of studies included in the meta-analysis.

\begin{tabular}{|c|c|c|c|c|c|c|c|}
\hline $\begin{array}{l}\text { Study, } \\
\text { location }\end{array}$ & $\begin{array}{l}\text { Patients } \\
\text { (n) }\end{array}$ & $\begin{array}{l}\text { Gender } \\
(\mathrm{M}: \mathrm{F})\end{array}$ & $\begin{array}{c}\text { Mean age } \\
\text { (years) }\end{array}$ & $\begin{array}{l}\text { Follow-up } \\
\text { time }\end{array}$ & $\begin{array}{l}\text { Characteristics } \\
\text { of the participants }\end{array}$ & Interventions & Outcomes analyzed \\
\hline $\begin{array}{l}\text { Jones } \\
1995 \text { (19), } \\
\text { UK }\end{array}$ & 63 & $24: 39$ & 70.5 & 6 months & $\begin{array}{l}\text { Bilateral knee } \\
\text { OA with bilateral } \\
\text { effusions }\end{array}$ & $\begin{array}{l}\text { Exp: } 32,20 \mathrm{mg} \\
\text { Hyalgan 5-weekly } \\
\text { injections; Ctl: } 31 \text {, } \\
20 \text { mg triamcinolone } \\
\text { hexacetonide single } \\
\text { injections followed } \\
\text { by } 4 \text { placebo injection }\end{array}$ & $\begin{array}{l}\text { VAS; duration of stiff- } \\
\text { ness; ROM; joint } \\
\text { effusion; local heat; } \\
\text { synovial thickening; } \\
\text { joint-line and peri- } \\
\text { articular tenderness }\end{array}$ \\
\hline
\end{tabular}

\begin{tabular}{|c|c|c|c|c|c|}
\hline $\begin{array}{l}\text { Frizziero } \\
2002(20), \\
\text { Italy }\end{array}$ & 99 & $46: 53$ & 49.5 & 6 months & $\begin{array}{l}\text { Kellgren-Lawrence } \\
\text { grades I-III; fulfilling } \\
\text { the clinical and radio- } \\
\text { logical criteria of the } \\
\text { American College } \\
\text { of Rheumatology (31) }\end{array}$ \\
\hline $\begin{array}{l}\text { Tasciotaoglu } \\
2003(21), \\
\text { Turkey }\end{array}$ & 60 & $0: 60$ & 59 & 6 months & $\begin{array}{l}\text { Kellgren-Lawrence } \\
\text { grade II-III knee } \\
\text { OA radiologically; } \\
\text { VAS of >40 mm }\end{array}$ \\
\hline
\end{tabular}

\begin{tabular}{|c|c|c|c|c|}
\hline $\begin{array}{l}\text { Caborn } \\
2004 \text { (23), }\end{array}$ & 218 & $95: 123$ & 63.1 & 26 weeks \\
\hline
\end{tabular}

USA

$\begin{array}{lllll}\text { Skwara } 2009 & 50 & 27: 23 & 61 & 12 \text { week } \\ \text { (Durolane) }(24), & & & \\ \text { Germany }\end{array}$

Kellgren-Lawrence grade II-III knee OA radiologically; VAS of $>40 \mathrm{~mm}$; persistent pain for $\geq 6$ months; Lequesne score $\geq 10$; good compliance grade II-III knee OA radiologically; VAS of $>40 \mathrm{~mm}$; persistent pain for $\geq 6$ months; Lequesne score $\geq 10$; good compliance

$\begin{array}{lllll}\text { Shimizu } & 51 & 13: 38 & >60 & 6 \text { months } \\ 2010(26), & & & \\ \text { Japan } & & & \end{array}$

OA findings on radiography and Kellgren-Lawrence grade II or III; persistent pain for $\geq 6$ months; hydro-; arthrosis; no treatment within 3 months
Exp: $52,2 \mathrm{ml}(20 \mathrm{mg})$

Hyalgan 5-weekly injections; Ctl: 47, $1 \mathrm{ml}$ (40 mg) methylprednisolone 3-weekly injections

Exp: 30, 2 ml (30 mg) Orthovisc 3-weekly injections; Ctl: $30,1 \mathrm{ml}$ (40 mg) methylprednisolone 3-weekly injections

Exp: 113, 2 ml (16 mg)

Synvisc 3-weekly injections; Ctl: 105, $2 \mathrm{ml}$ (40 mg) triamcinolone hexacetonide single injection

Exp: 24, $3 \mathrm{ml}$ (60 mg)

Durolane single injection; Ctl: 26, $1 \mathrm{ml}$ $(10 \mathrm{mg})$ triamcinolone single injection

VAS; Lequesne index; functional index; range of knee lexion; adverse events

\section{WOMAC scores;} VAS

Gait pattern (ROM of knee and hip); muscle activity; VAS;

Lequesne index; KSS; SF-36
VAS; duration of stiff-
ness; ROM; joint
effusion; local heat;
synovial thickening;
joint-line and peri-
articular tenderness
Arthroscopic findings;
VAS; morning stiff-
ness; maximum active
extension and flexion

VAS; duration of stiff-
ness; ROM; joint
effusion; local heat;
synovial thickening;
joint-line and peri-
articular tenderness
Arthroscopic findings;
VAS; morning stiff-
ness; maximum active
extension and flexion

VAS; duration of stiff-
ness; ROM; joint
effusion; local heat;
synovial thickening;
joint-line and peri-
articular tenderness
Arthroscopic findings;
VAS; morning stiff-
ness; maximum active
extension and flexion

VAS; duration of stiff-
ness; ROM; joint
effusion; local heat;
synovial thickening;
joint-line and peri-
articular tenderness
Arthroscopic findings;
VAS; morning stiff-
ness; maximum active
extension and flexion
Exp: 21,2 ml (20 mg) Gait pattern; muscle Ostenil 5-weekly activity; VAS;
injections; Ctl: 21, $1 \mathrm{ml}$ Lequesne index; KSS;
$(10 \mathrm{mg})$ triamcinolone SF-36
5-weekly injections
Exp: 26, 25 mg Na-HA 5 -weekly injections; Ctl: 25, 4 mg Decadron single injection
Pain/inflammation scores; VAS; joint fluid levels (HA, MMP-9, TIMP-1); Gotoh score

\footnotetext{
Exp, experimental group; Ctl, control group; VAS, visual analog score; ROM, range of motion; HA, hyaluronic acid; MMP, matrix metalloproteinases; TIMP, tissue inhibitors of metalloproteinases; WOMAC, Western Ontario and McMaster osteoarthritis index; OA, osteoarthritis; M, male; F, female; SF-36, 36-Item Short Form Health Survey; KSS, Knee Society Clinical Rating System. The number after Exp and Ctl refers to the number of patients in each group.
}

A total of five studies (23-25,27,30), including $217 \mathrm{HA}$ and 194 CS-treated patients $(n=411)$, reported detailed data on the VAS score of knee OA after six months. HA injections were found to significantly reduce the VAS score by a mean of $9.01 \mathrm{~mm}$, ranging between 5.40 and 12.62, when compared with that in the CS group (95\% CI; P<0.00001; $\mathrm{I}^{2}=47 \%$; Fig. 2). 


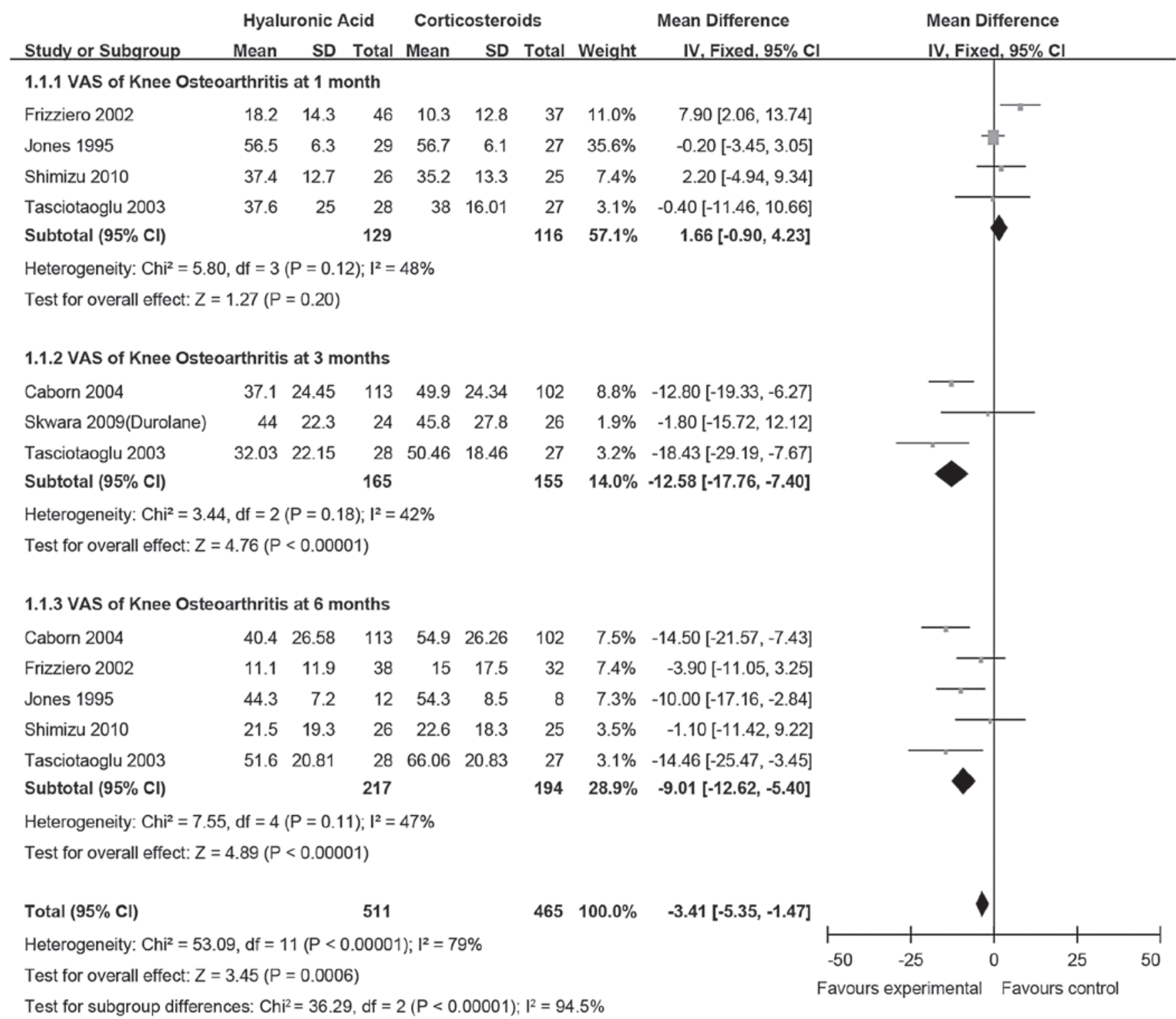

Figure 2. Forest plot diagram showing the VAS of knee OA. VAS, visual analog score; SD, standard deviation; CI, confidence interval; OA, osteoarthritis.

Lequesne index of knee $O A$. Three studies $(25,28,29)$, including $72 \mathrm{HA}$ and $68 \mathrm{CS}$-treated patients $(\mathrm{n}=140)$, reported detailed data on the Lequesne index of knee OA after three months. No statistically significant differences in the Lequesne index were observed between the two groups (mean difference (MD), -0.50; 95\% CI, -1.91, 0.91; $\mathrm{P}=0.48 ; \mathrm{I}^{2}=75 \%$; Fig. 3).

KSS of knee OA. Two studies $(28,29)$, including $44 \mathrm{HA}$ and 41 CS-treated patients $(\mathrm{n}=85)$, reported detailed data on the KSS of knee OA after three months. No statistically significant differences in the KSS were identified between the two groups (MD, -6.09; 95\% CI, -14.52, 2.33; $\mathrm{P}=0.16 ; \mathrm{I}^{2}=0 \%$; Fig. 4).

Maximum flexion of knee OA. Three studies $(25,28,29)$, including $72 \mathrm{HA}$ and $68 \mathrm{CS}$-treated patients $(\mathrm{n}=140)$, reported detailed data on the maximum flexion of knee OA after three months of treatment. No statistically significant differences in the maximum flexion were observed between the two groups (MD, 0.61; 95\% CI, -1.36, 2.59; $\mathrm{P}=0.54 ; \mathrm{I}^{2}=0 \%$; Fig. 5).

Adverse events of knee OA. In total, three studies $(24,25,27)$, including $171 \mathrm{HA}$ and 144 CS-treated patients $(\mathrm{n}=315)$, reported detailed data on the adverse events of knee OA.
No statistically significant differences in the adverse events were observed between the two groups (RR, 0.77; 95\% CI, $0.54,1.12 ; \mathrm{P}=0.17 ; \mathrm{I}^{2}=0 \%$; Fig. 6 ).

Publication bias analysis. The VAS of knee OA was used for the funnel plot analysis of publication bias (Fig. 7), which revealed there was no marked publication bias evident for the outcome.

\section{Discussion}

In the present study, a meta-analysis of trials comparing HA with CS for the treatment of OA was performed, and the results indicated that the curative effect of the different treatments varied over time. With regard to the VAS of knee OA, the two drugs (HA and CS) appeared to be equally effective for pain in the short term ( $\leq 1$ month). However, after $\geq 3$ months, HA was found to have a greater relative effect compared with CS. By contrast, for other indicators, including the Lequesne index, KSS and maximum flexion, no statistically significant differ- 


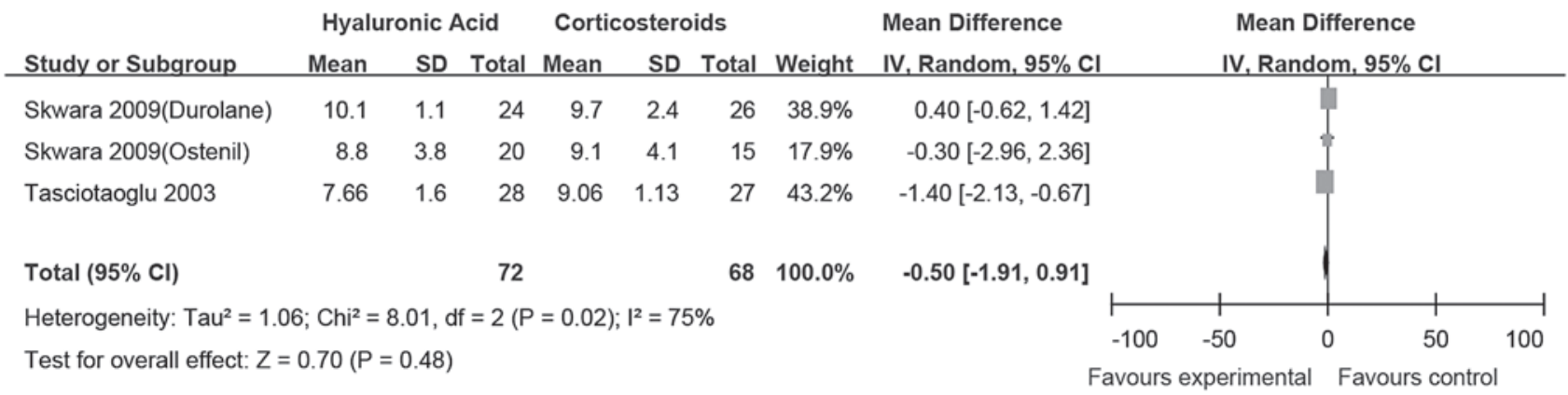

Figure 3. Forest plot diagram showing the Lequesne index of knee OA. SD, standard deviation; CI, confidence interval; OA, osteoarthritis.

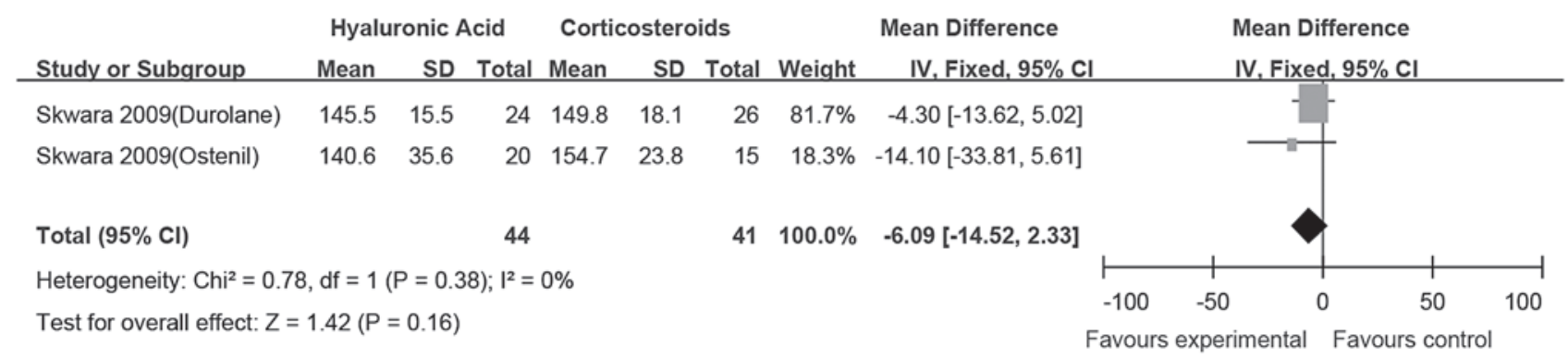

Figure 4. Forest plot diagram showing the KSS of knee OA. SD, standard deviation; CI, confidence interval; KSS, Knee Society Clinical Rating Score; OA, osteoarthritis.

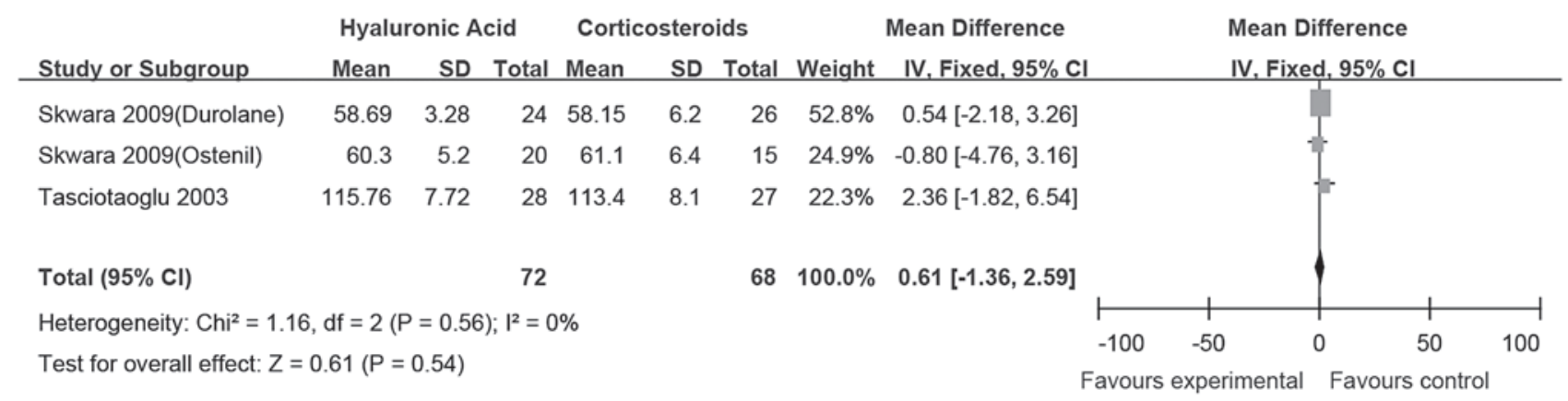

Figure 5. Forest plot diagram showing the maximum flexion of knee OA. SD, standard deviation; CI, confidence interval; OA, osteoarthritis.

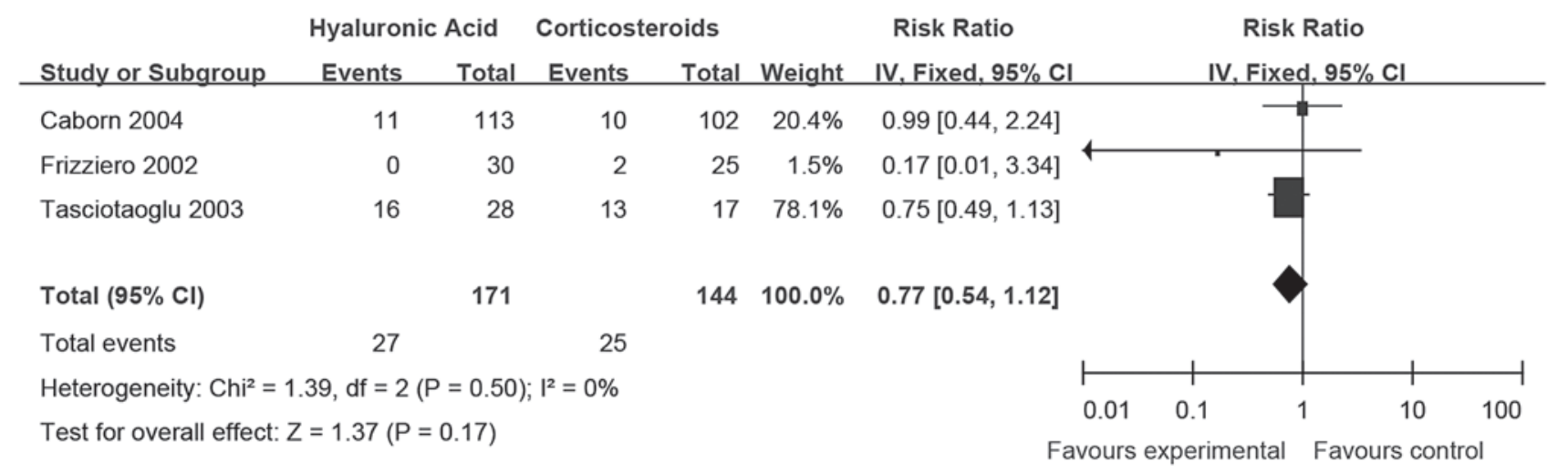

Figure 6. Forest plot diagram showing the adverse events of knee OA. SD, standard deviation; CI, confidence interval; OA, osteoarthritis.

ences were observed between the two treatment approaches for knee OA. Similarly, for the adverse reactions, no difference was observed between the two drugs. However, this meta-anal- ysis compared the two interventions on their efficacy in knee OA treatment and did not directly compare their efficacy with a placebo; thus, it is important to determine whether HA and 


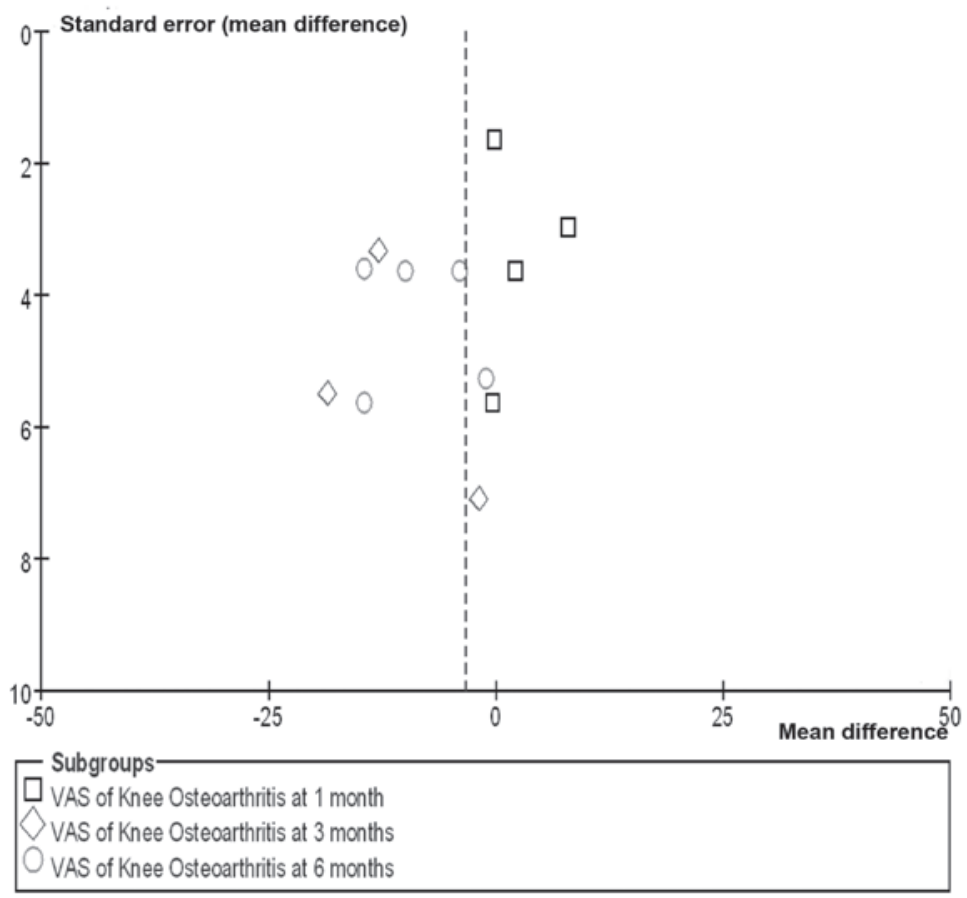

Figure 7. Funnel plot showing the publication bias of the subgroups with regard to VAS at the different time periods. VAS, visual analog score. SE, standard error; MD, mean difference.

CS are effective in the treatment of OA according to recent studies $(2,16,20,35)$. The results indicate that the therapeutic effect of HA may be longer lasting compared with CS.

Bannuru et al (15) also performed a meta-analysis of systematic reviews analyzing the efficacy of intra-articular $\mathrm{HA}$ in the treatment of OA compared with intra-articular CS. However, only VAS was used as an evaluation index and all the trials included were published prior to 2004. Despite these differences, the conclusions of the meta-analysis were in accordance with the results of the present study.

One unique feature of the present meta-analysis was that the therapeutic response was based on time by separately pooling the data for each time point. The pattern of the therapeutic response was then attributed to different interventions to a large extent. However, not all the trials had the full clinical data associated with each of the time points. Therefore, the data made available were collected for comparative analysis. Other similar prospective RCTs exist; however, the complete data were not available.

In order to minimize publication bias, a large search strategy was used independently by two reviewers. The funnel plot (Fig. 7) indicated that there was no marked publication bias with regard to the selection of trials included in the meta-analysis. The reliability on the risk of bias assessment and data extraction was enhanced as the two authors performed the procedures independently, from which a consensus was then obtained. In addition, the bias was minimized through study design and quality by performing sensitivity analyses based on the study design, which provided an overview of the risk of bias assessment.

An additional problem may have been the use of Jadad scores to evaluate the quality of the trials. The majority of the studies included in the meta-analysis had Jadad scores greater than three, and a number had full Jadad scores, indicating that the majority of the articles were of high quality. However, the Jadad scoring system has been described as simplistic since it takes into account a limited number of variables and does not consider bias as a result of allocation concealment (36). Therefore, the Pedro format (www.cchs.usyd.edu.au/pedro/), which includes more variables, was used to further assess the quality of the trials.

The safety of the two interventions was also investigated. The majority of the injections were performed at an injection site above the tibial plateau and lateral to the patellar tendon, with the knee flexed at $\sim 90^{\circ}$ and without ultrasound or fluoroscopy guidance. Notably, the adverse effects were rare or insignificant $(37,38)$, and the occurrence rate was not significantly different between the two interventions. The most common adverse effects were arthralgia, injection site pain, joint swelling and injection site edema (27). No joint space loss was observed at the knee joint following interventions in OA; thus, we hypothesize that clinical operators should be careful when the injections are performed in order to alleviate the discomfort of the procedure, localized pain post-injection and flushing.

There were certain limitations in the present. Firstly, the number of included trials was limited, which may have resulted in insufficient significant effectiveness. In addition, the meta-analysis suffered from the pooling of a variety of HA agents that differed with regard to characteristics, including molecular weight, origin, viscosity and cross-linking. To avoid this type of bias, sensitivity analyses may be performed. However, sensitivity analysis based on the viscosity or molecular weights was not performed since this may have biased the review as a direct comparison between different agents. Furthermore, sensitivity analyses based on comparing the same type of HA agent with one type of CS agent were not successful, primarily due to the paucity of the data. 
In conclusion, the results of the meta-analysis demonstrated that HA has a similar level of pain relief compared with $\mathrm{CS}$ in the short term (up to one month); however, HA is more effective than CS over a longer time period (up to six months). The potential for adverse events are similar between the two interventions. Understanding the length of the clinical efficacy and adverse events of these two drugs is useful for clinicians to produce a therapeutic regimen for OA patients. However, more high-quality RCTs with long-term follow-ups and large sample sizes are required in the future.

\section{Acknowledgements}

The authors thank Zirui Yu from the Orthopedics Department, Second Affiliated Hospital of Xi'an Jiaotong University (Xi'an, China) for his assistance in searching the databases, giving critics on the manuscript content and revising the manuscript.

\section{References}

1. Poole AR, Rizkalla G, Ionescu M, et al: Osteoarthritis in the human knee: a dynamic process of cartilage matrix degradation, synthesis and reorganization. Agents Actions Suppl 39: 3-13, 1993.

2. Ringdahl E and Pandit S: Treatment of knee osteoarthritis. Am Fam Physician 83: 1287-1292, 2011.

3. Hootman JM and Helmick CG: Projections of US prevalence of arthritis and associated activity limitations. Arthritis Rheum 54: 226-229, 2006

4. Mounsey A and Ewigman B: Arthroscopic surgery for knee osteoarthritis? Just say no. J Fam Pract 58: 143-145, 2009.

5. Day R, Brooks P, Conaghan PG, et al: A double blind, randomized, multicenter, parallel group study of the effectiveness and tolerance of intraarticular hyaluronan in osteoarthritis of the knee. J Rheumatol 31: 775-782, 2004.

6. Shimazu A, Jikko A, Iwamoto M, et al: Effects of hyaluronic acid on the release of proteoglycan from the cell matrix in rabbit chondrocyte cultures in the presence and absence of cytokines. Arthritis Rheum 36: 247-253, 1993.

7. Gotoh S, Onaya J, Abe M, et al: Effects of the molecular weight of hyaluronic acid and its action mechanisms on experimental joint pain in rats. Ann Rheum Dis 52: 817-822, 1993.

8. Asari A, Miyauchi S, Matsuzaka S, et al: Molecular weight-dependent effects of hyaluronate on the arthritic synovium. Arch Histol Cytol 61: 125-135, 1998.

9. Rutjes AW, Jüni P, da Costa BR, et al: Viscosupplementation for osteoarthritis of the knee: a systematic review and meta-analysis Ann Intern Med 157: 180-191, 2012.

10. Campbell J, Bellamy $\mathrm{N}$ and Gee T: Differences between systemmatic reviews/meta-analyses of hyaluronic acid/hyaluronan/hylan in osteoarthritis of the knee. Osteoarthritis Cartilage 15: 1424-1436, 2007.

11. Arrich J, Piribauer F, Mad P, et al: Intra-articular hyaluronic acid for the treatment of osteoarthritis of the knee: systematic review and meta-analysis. CMAJ 172: 1039-1043, 2005

12. Lo GH, LaValley M, McAlindon T and Felson DT: Intraarticular hyaluronic acid in the treatment of knee osteoarthritis: a meta-analysis. JAMA 290: 3115-3121, 2003.

13. Medina JM, Thomas A and Denegar CR: Knee osteoarthritis: should your patient opt for hyaluronic acid injection? J Fam Pract 55: 669-675, 2006.

14. Creamer P: Intra-articular corticosteroid treatment in osteoarthritis. Curr Opin Rheumatol 11: 417-421, 1999.

15. Bannuru, RR, Natov NS, Obadan IE, et al: Therapeutic trajectory of hyaluronic acid versus corticosteroids in the treatment of knee osteoarthritis: a systematic review and meta-analysis. Arthritis Rheum 61: 1704-1711, 2009.

16. Colen S, van den Bekerom MP, Mulier M and Haverkamp D: Hyaluronic acid in the treatment of knee osteoarthritis: a systematic review and meta-analysis with emphasis on the efficacy of different products. BioDrugs 26: 257-268, 2012.
17. Bannuru RR, Natov NS, Dasi UR, et al: Therapeutic trajectory following intra-articular hyaluronic acid injection in knee osteoarthritis - meta-analysis. Osteoarthritis Cartilage 19: 611-619, 2011.

18. Divine JG, Zazulak BT and Hewett TE: Viscosupplementation for knee osteoarthritis: a systematic review. Clin Orthop Relat Res 455: 113-122, 2007.

19. Arroll B and Goodyear-Smith F: Corticosteroid injections for osteoarthritis of the knee: meta-analysis. BMJ 328: 869, 2004.

20. Godwin $M$ and Dawes M: Intra-articular steroid injections for painful knees. Systematic review with meta-analysis. Can Fam Physician 50: 241-248, 2004.

21. Jadad AR, Moore RA, Carroll D, et al: Assessing the quality of reports on randomised clinical trials: is blinding necessary? Control Clin Trials 17: 1-12, 1996.

22. Higgins JP and Thompson SG: Quantifying heterogeneity in a meta-analysis. Stat Med 21: 1539-1558, 2002.

23. Jones AC, Pattrick M, Doherty S and Doherty M: Intra-articular hyaluronic acid compared to intra-articular triamcinolone hexacetonide in inflammatory knee osteoarthritis. Osteoarthritis Cartilage 3: 269-273, 1995.

24. Frizziero L and Pasquali Ronchetti I: Intra-articular treatment of osteoarthritis of the knee: an arthroscopic and clinical comparison between sodium hyaluronate $(500-730 \mathrm{kDa})$ and methylprednisolone acetate. J Orthop Traumatol 3: 89-96, 2002.

25. Tasciotaoglu F and Oner C: Efficacy of intra-articular sodium hyaluronate in the treatment of knee osteoarthritis. Clin Rheumatol 22: 112-117, 2003.

26. Leopold SS, Redd BB, Warme WJ, et al: Corticosteroid compared with hyaluronic acid injections for the treatment of osteoarthritis of the knee. A prospective, randomized trial. J Bone Joint Surg Am 85-A: 1197-1203, 2003.

27. Caborn D, Rush J, Lanzer W, et al; Synvisc 901 Study Group: A randomized, single-blind comparison of the efficacy and tolerability of hylan G-F 20 and triamcinolone hexacetonide in patients with osteoarthritis of the knee. J Rheumatol 31: 333-343, 2004.

28. Skwara A, Ponelis R, Tibesku CO, et al: Gait patterns after intraarticular treatment of patients with osteoarthritis of the knee - hyaluronan versus triamcinolone: a prospective, randomized, doubleblind, monocentric study. Eur J Med Res 14 157-164, 2009.

29. Skwara A, Peterlein CD, Tibesku CO, et al: Changes of gait patterns and muscle activity after intraarticular treatment of patients with osteoarthritis of theknee: a prospective, randomised, doubleblind study. Knee 16: 466-472, 2009.

30. Shimizu M, Higuchi H, Takagishi K, et al: Clinical and biochemical characteristics after intra-articular injection for the treatment of osteoarthritis of the knee: prospective randomized study of sodium hyaluronate and corticosteroid. J Orthop Sci 15: 51-56, 2010.

31. Hochberg MC, Altman RD, April KT, et al: American College of Rheumatology 2012 recommendations for the use of nonpharmacologic and pharmacologic therapies in osteoarthritis of the hand, hip, and knee. Arthritis Care Res (Hoboken) 64: 465-474, 2012 .

32. de Campos GC, Rezende MU, Pailo AF, et al: Adding triamcinolone improves viscosupplementation: a randomized clinical trial. Clin Orthop Relat Res 471: 613-620.

33. Ozturk C, Atamaz F, Hepguler S, et al: The safety and efficacy of intraarticular hyaluronan with/without corticosteroid in knee osteoarthritis: 1-year, single-blind, randomized study. Rheumatol Int 26: 314-319, 2006.

34. Tekeoglu I, Adak B, Goksoy T and Tosun N: Effects of intra-articular injections of sodium hyaluronate (Orthovisc) and betamethasone on osteoarthritis of the knee. J Rheum Med Rehab 9: 220-224, 1998.

35. Bellamy N, Campbell J, Robinson V, et al: Intraarticular corticosteroid for treatment of osteoarthritis of the knee. Cochrane Database Syst Rev: CD005328, 2006.

36. Berger VW and Alperson SY: A general framework for the evaluation of clinical trial quality. Rev Recent Clin Trials 4: 79-88, 2009.

37. Cheng $J$ and Abdi S: Complications of joint, tendon and muscle injections. Tech Reg Anesth Pain Manag 11: 141-147, 2007.

38. Habib GS, Saliba W and Nashashibi M: Local effects of intra-articular corticosteroids. Clin Rheumatol 29: 347-356, 2010. 